\title{
Fibula Bone Graft Supporting Failed Posterior Rod Implants in Thoracic Spine Tuberculosis: A Case Report
}

\section{Muath Mamdouh Mahmod Al-Chalabi ${ }^{1^{*}}$ (D) and Wan Azman Wan Sulaiman ${ }^{2}$}

${ }^{1}$ Reconstructive Sciences Unit, Universiti Sains Malaysia, Malaysia

${ }^{2}$ Head Department of Reconstructive Sciences Unit, Universiti Sains Malaysia, Malaysia

*Corresponding author: Dr. Muath Mamdouh Mahmod Al-Chalabi, Reconstructive Sciences Unit, PPSP Health Campus, Universiti Sains Malaysia, Kubang Kerian, 16150 Kelantan, Malaysia, Tel: 00601139181101

\begin{abstract}
Tuberculosis of the spine is a challenging illness to treat because of the prolonged time of conservative treatment and the technical difficulties of surgical intervention. The disease remains a significant public health issue in developing countries but is also returning in developed countries due to immigration and particularly in immune-compromised patients. The most common symptom reported is back pain, and the most common segment involved; is the thoracic spine.

One of the essential options for treating spinal tuberculosis is a surgical intervention that comprises bone grafting. The main aims of surgical management of spinal tuberculosis comprise debridement of affected vertebrae or affected bone, decompression of spinal cord, correction of deformities caused by tuberculosis, stabilization of the spine, and more protection of the spinal cord. Neurological involvement due to Pott's disease is quite benign if urgent decompression is achieved at the onset of the disease.

Effective stabilization is indicated to offer mechanical stability in a spinal tuberculosis lesion, but many complications are still anticipated. One of the challenging complications; is implant (Rod) breakage, which may arise shortly after the operation.

We report a case of young female 23-years-old, who presented with tubercles thoracic vertebral bone defects, vertebral bone fusion, and spondylodiscitis. We conclude that performing of fibula bone allograft, which placed along sides of the affected vertebrae, concurrently with filling a defected bone by pieces of an allograft, prevents the breakage of implants by giving firm base which can hold and support Rod and Screw.
\end{abstract}

\section{Keywords}

Bone tuberculosis, Spine TB, Fibula bone graft

\section{Introduction}

Bone and joint tuberculosis (TB) is an ancient disease as there is evidence of osteoarticular TB has been detected in Egyptian mummies, Iron Age remains from Asia and skeletons of Europeans living in the Middle Ages. The true incidence of vertebral TB worldwide is still unknown, but it has increased in the last decade in developed countries with immigration and the HIV epidemic [1]. Percival Pott in 1779 was the first one to present the definitive modern description of spinal deformity and paraplegia, resulting in spinal TB; from this time, spinal TB was called "Pott's Disease" [2].

Diagnosis of spinal TB is difficult, and it is commonly presented at an advanced stage. Delays in establishing diagnosis and management contribute to higher rates of complications such as spinal cord compression, spinal deformity, and spondylodiscitis which refers to an infection affecting the intervertebral disk, the vertebral body or the posterior arch of the vertebra due to pyogenic, granulomatous (tuberculosis, brucellosis, or fungal infection) or parasitic infestation [3]. Patients mostly present with lower limb weakness. Usually, patients treated according to anti-TB drug protocol once TB diagnosed. In the case of extrapulmonary TB (e.g., spinal TB), some extra treatment options should be followed starting from conservative management such as immobilization using body casts or plaster beds.

Surgical treatment choices exist for different presentations to include anterior spinal fusion, anterior-posterior spinal fusion, posterior spinal fusion alone, and pos-

Citation: Al-Chalabi MMM, Sulaiman WAW (2020) Fibula Bone Graft Supporting Failed Posterior Rod Implants in Thoracic Spine Tuberculosis: A Case Report. Int Arch Orthop Surg 3:021. doi. org/10.23937/2643-4016/1710021

Accepted: September 23, 2020; Published: September 25, 2020

Copyright: (C) 2020Al-Chalabi MMM, et al. This is an open-access article distributed under the terms of the Creative Commons Attribution License, which permits unrestricted use, distribution, and reproduction in any medium, provided the original author and source are credited 
terior fusion followed by anterior spinal fusion [4]. The standard surgical protocols include vertebrectomy and debridement, nerve decompression, and reconstruction of the spinal alignment with artificial internal implants. Reconstruction of the normal vertebral position is most challenging. Surgical reconstruction must provide sufficient and lasting mechanical strength and spinal stability [5]. We reported a case of thoracic spine TB complicated with neurological manifestations throughout two months' duration. We manage here surgically in combination with the orthopedic spine surgical team to perform fibula bone graft to give a base supporting and fixing posterior spinal rod implants.

\section{Case Report}

Ms. N. is a 23-years-old Malay female known case of thoracic spine TB complicated with thoracic vertebral bone defects, vertebral bone fusion and spondylodiscitis. Over a short period of duration, clinical manifestations progressed.

The condition started shortly before admission when the patient complained of pain confined to the posterior cervical and thoracic area. The pain was aggravated by movement and relieved by lying on the bed. No other associated signs and symptoms. Subsequent investigations performed in Hospital Universiti Sains Malaysia (HUSM) which include:

- Hematology blood profile and serum electrolytes were negative except slightly increased ESR.

- Bacteriology/Mycology (Thoracic spine tissue for growth, AFB) all were negative.

- Serology (Hepatitis B and C, Syphilis, HIV) all were negative.

- Immunology CRP was positive, which controlled and returned negative after introducing of antibiotics.
- Chest X-ray was normal.

- Cervical-Thoracic spine X-ray (showed lytic lesions in two thoracic vertebral bodies).

- MRI (showed collapsed fused vertebral body of thoracic vertebrae T2-T3 and spinal cord compression at the same level), as shown in Figure 1.

The diagnosis was TB spondylodiscitis complicated with spinal cord compression. Anti-TB therapy immediately started, and the subsequent plan was debridement, spinal cord decompression and surgical instrument implantation. Rod implants placed to support vertebrae from C7-T5 as shown in Figure 2. After one month, the patient again came complaining of back and posterior neck pain at the site of operation without any history of trauma. An X-ray showed broken right implanted Rod at a level of T2-T3 as shown in Figure 3. The second operation performed to revise and replace the broken right Rod implant. The patient followed up by X-ray; all views were acceptable. One month later, the patient came again complaining of severe back pain.

\section{On Examination}

All vitals and examinations were normal, even neurological examination (power, sensation and reflexes) but the patient in back pain at the operation site. Anti-TB medications were ongoing. CT showed a broken left Rod implant as in Figure 4.

After considering broke both Rod implant in different occasion, we plan for supporting reconstruction by fibula bone graft placed on both sides were the Rods implanted to give firm base which can hold and support Rod and Screw.

The donor site (left leg) was planned preoperatively, with a fibular length of $17 \mathrm{~cm}$. Both proximal fibular end $(7 \mathrm{~cm})$ and distal fibular end $(8 \mathrm{~cm})$ left intact as in

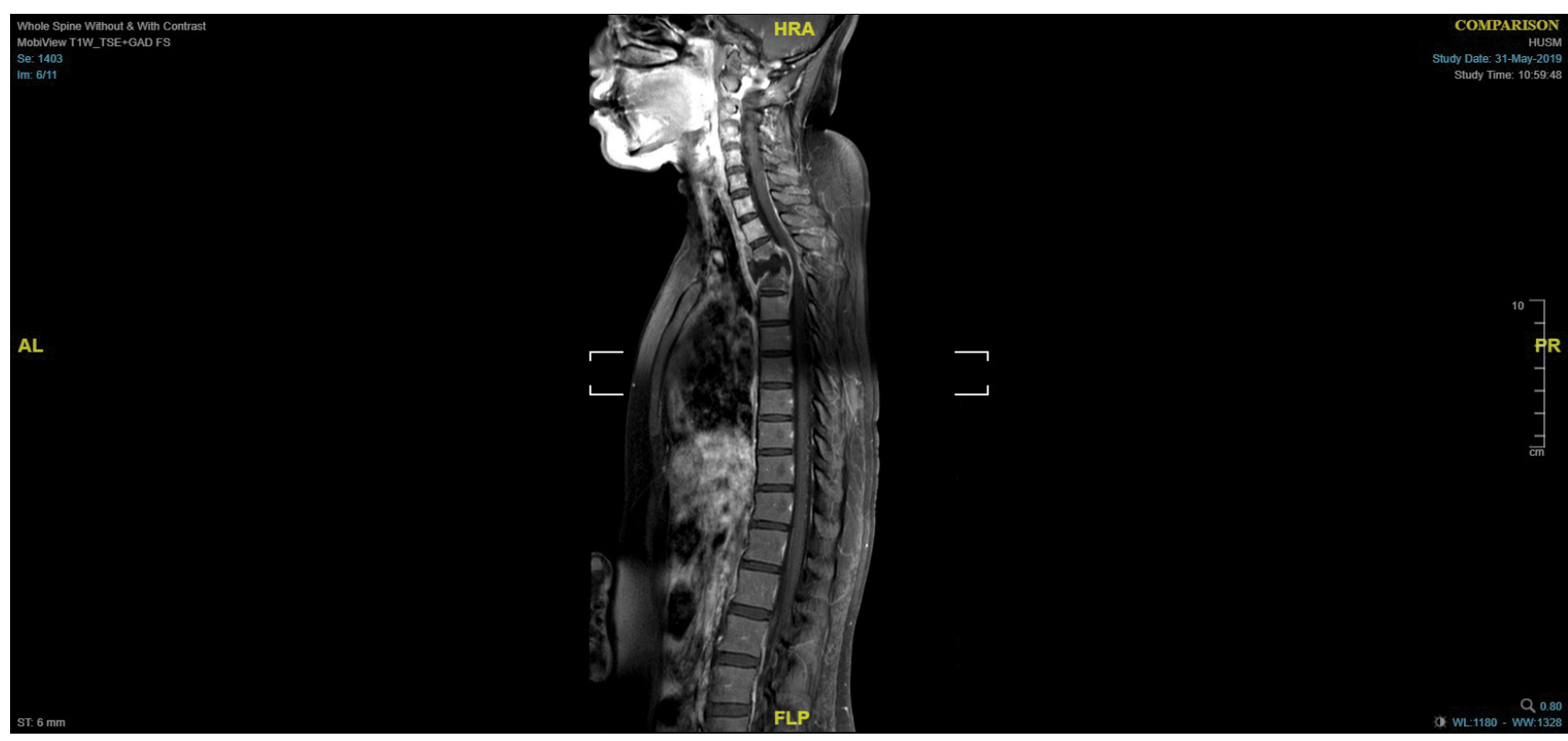

Figure 1: MRI with contrast showing collapsed, fused thoracic vertebrae (T2-T3) with spinal cord compression. 


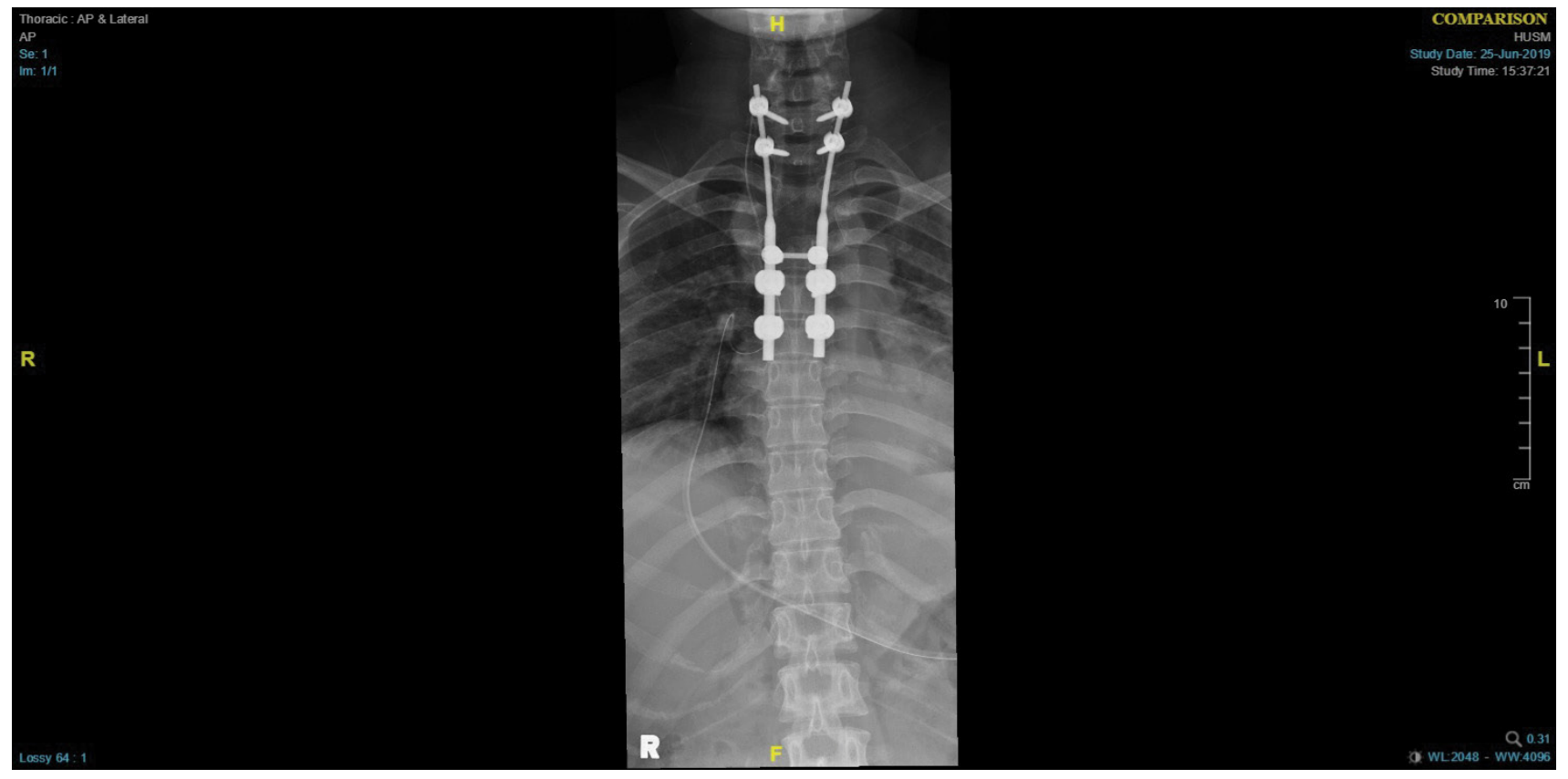

Figure 2: Supine AP chest X-ray showing Rod implants on both vertebral sides (C7-T5).

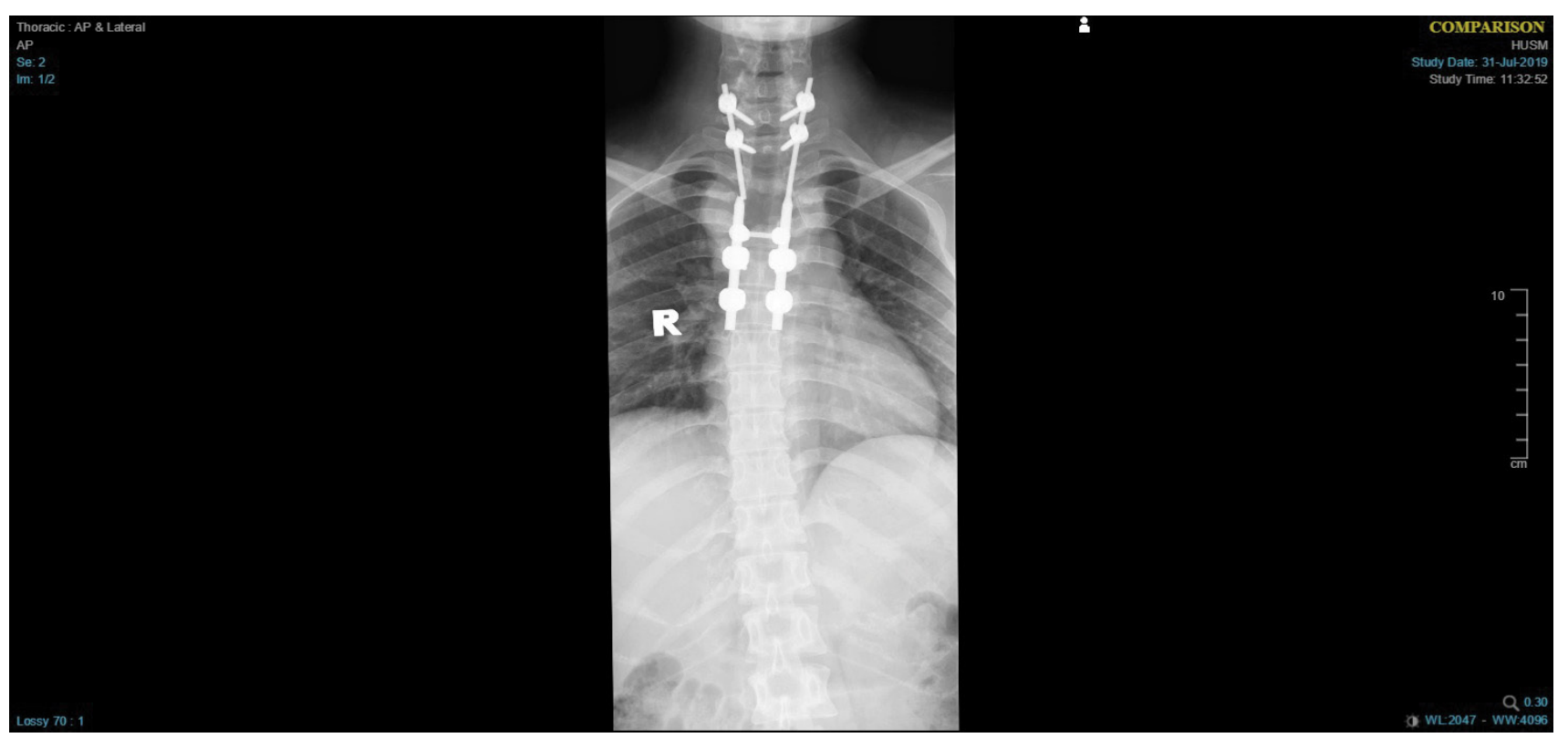

Figure 3: AP thoracic X-ray showing the broken right Rod implant.

Figure 5. Intra-operatively patient positioned in prone with left leg semi flexed, longitudinal incision posterior to fibula bone performed. Intermuscular septum identified and separated, then $17 \mathrm{~cm}$ of fibular bone graft harvested. Muscles and fascia closed and drain placed. The dermal layer closed in layers and skin sutured. Harvested fibula bone split longitudinally into two pieces as shown in Figure 6 to be suitable for both affected vertebral spine sides. After approaching the targeted area in the back through a surgical incision, each piece of fibula bone was placed lateral to transition Rod for extra support.

Wound inspection performed on day three postoperatively and was good without any complications. Pain controlled and Sternal Occipital Mandibular Immobilizer (SOMI) brace requested to restrict movement. Follow up continued for ten days, and all wounds were dry and intact without any complaint. X-ray taken post-operatively to confirm stability of Rods as shown in Figure 7 and the patient discharged with a given $\mathrm{X}$-ray appointment to reassess the graft and implant.

\section{Postoperative Follow-Up}

Seven months follow-up reflected well-fixed implanted rode after strengthen it by fibula bone graft. The patient's general condition improved, and all complaints resulted from previously broken rods subsided with no new other complaints. Figure 8 showing three months post-operative X-ray view and Figure 9 showing 


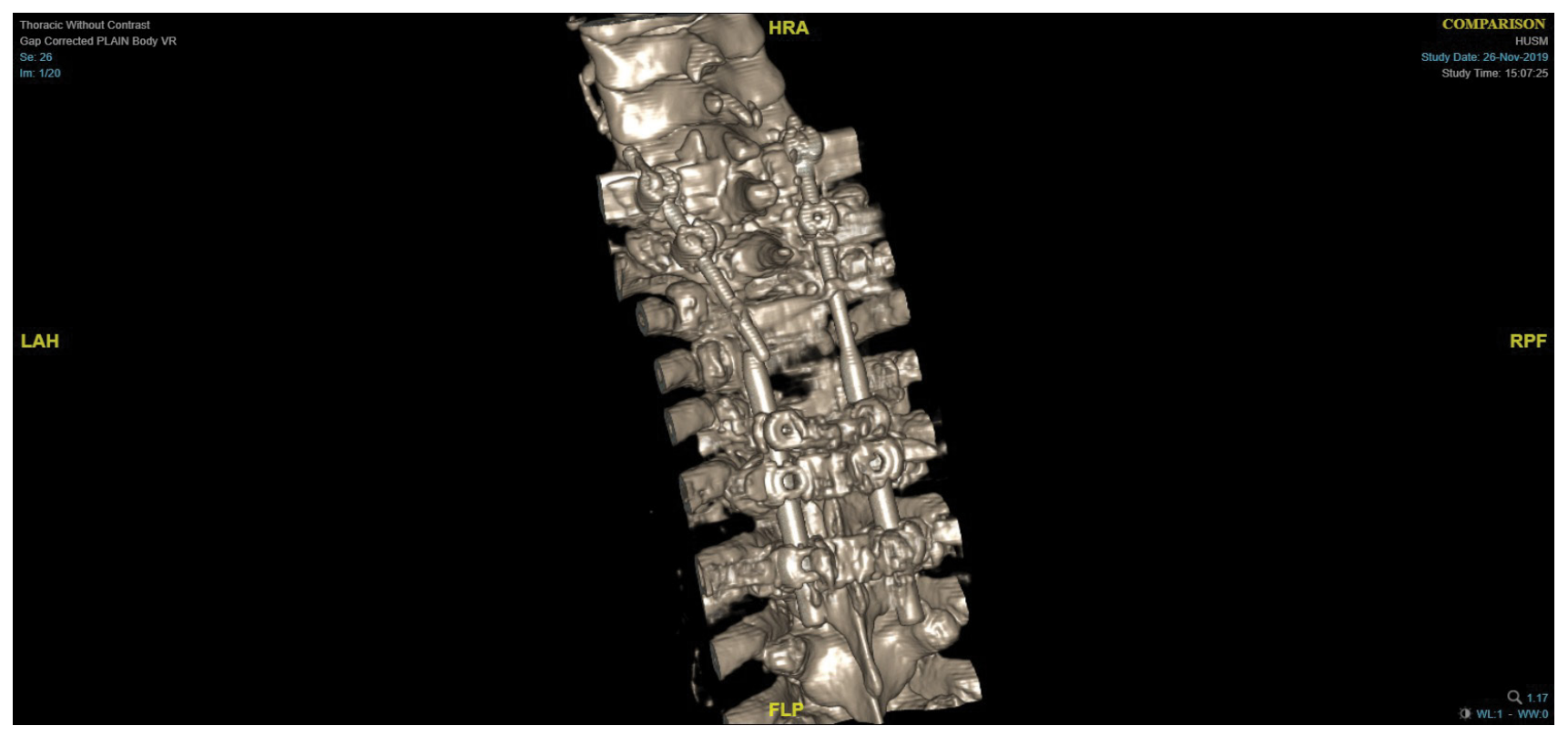

Figure 4: Three dimensional CT showing broken left Rod implant and bone defect.

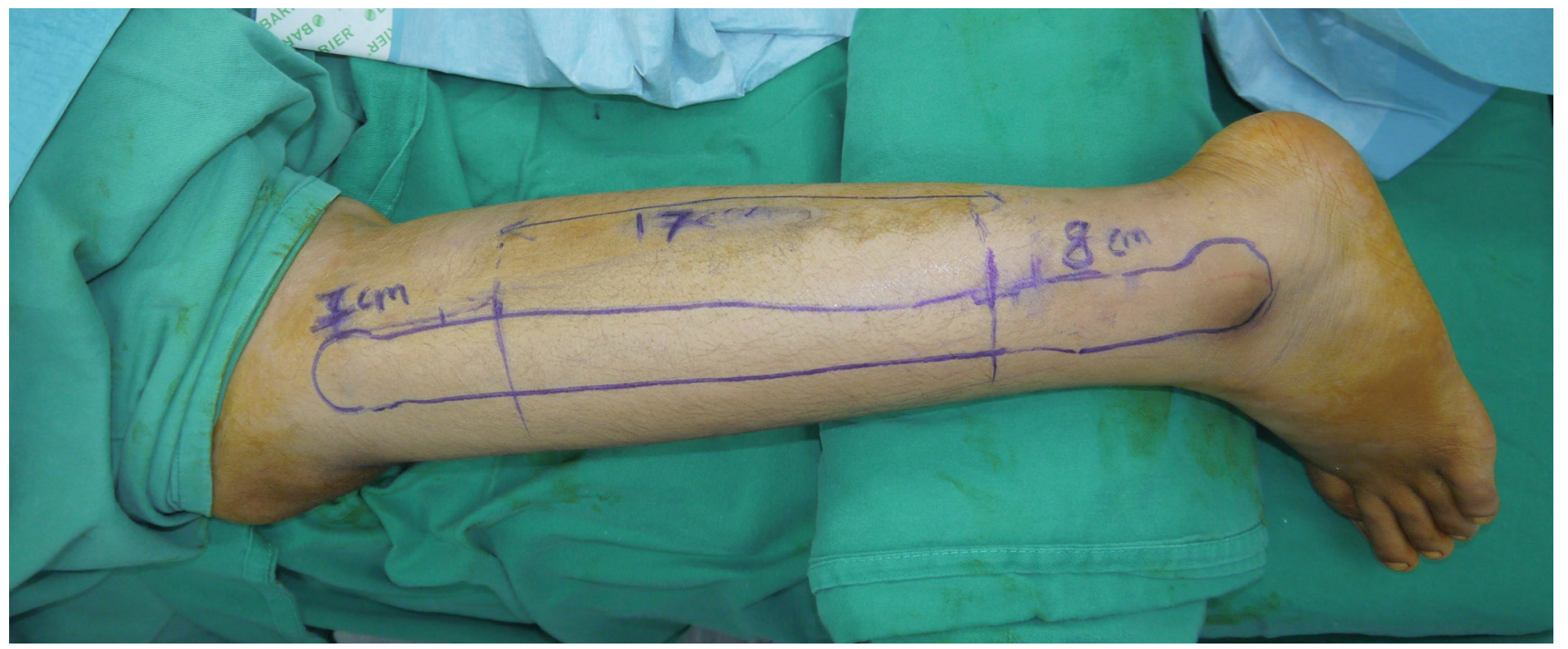

Figure 5: Preoperative planning for fibula bone graft.

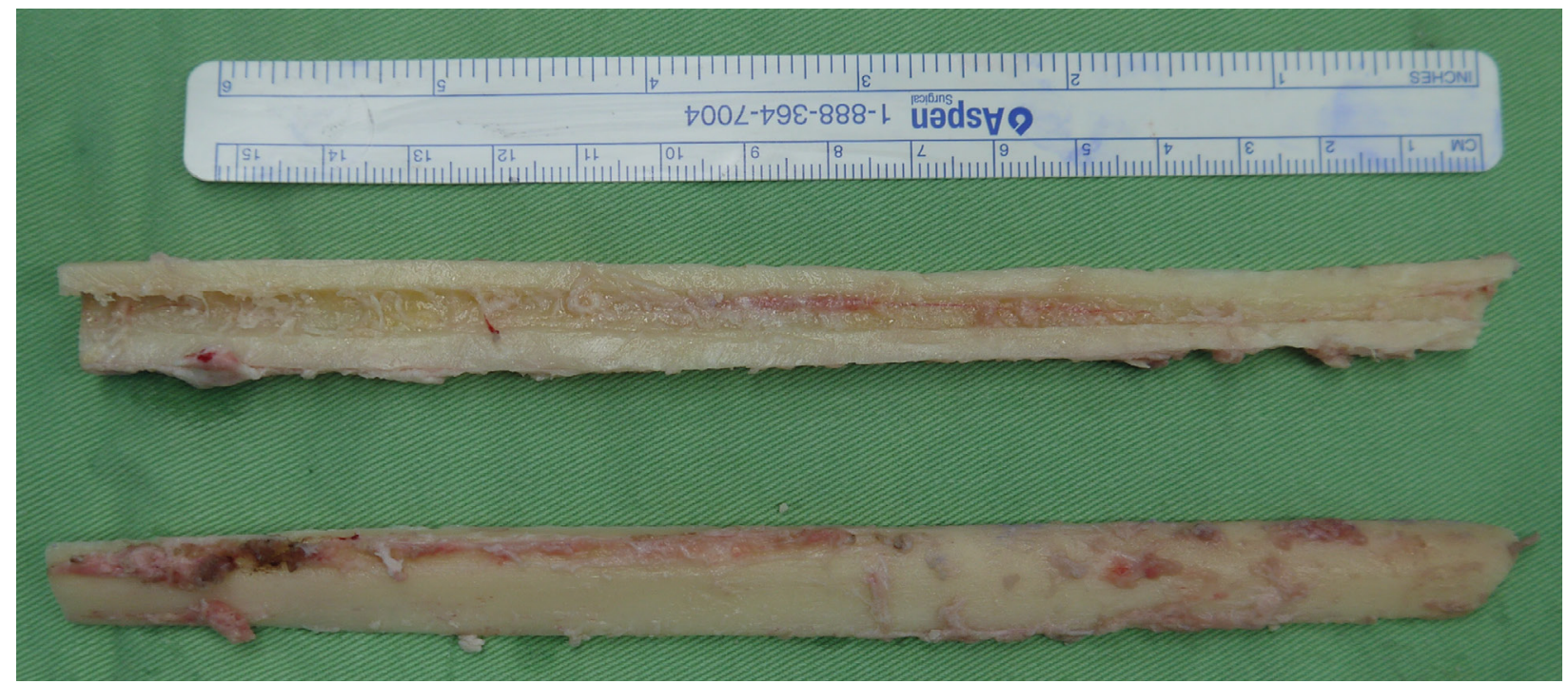

Figure 6: Splitted fibula bone graft. 


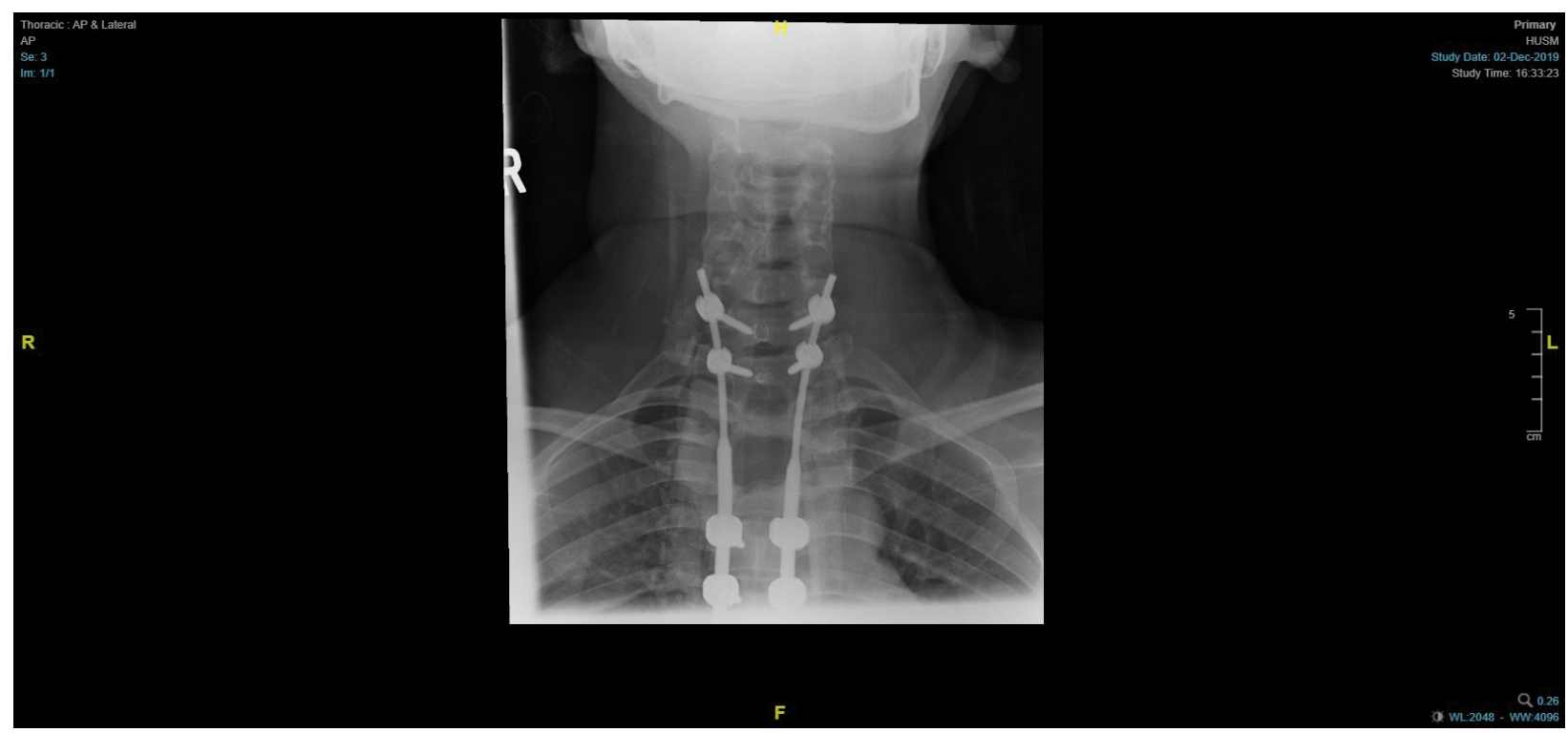

Figure 7: Post-operative AP thoracic X-ray showing Rod implants on both vertebral sides.

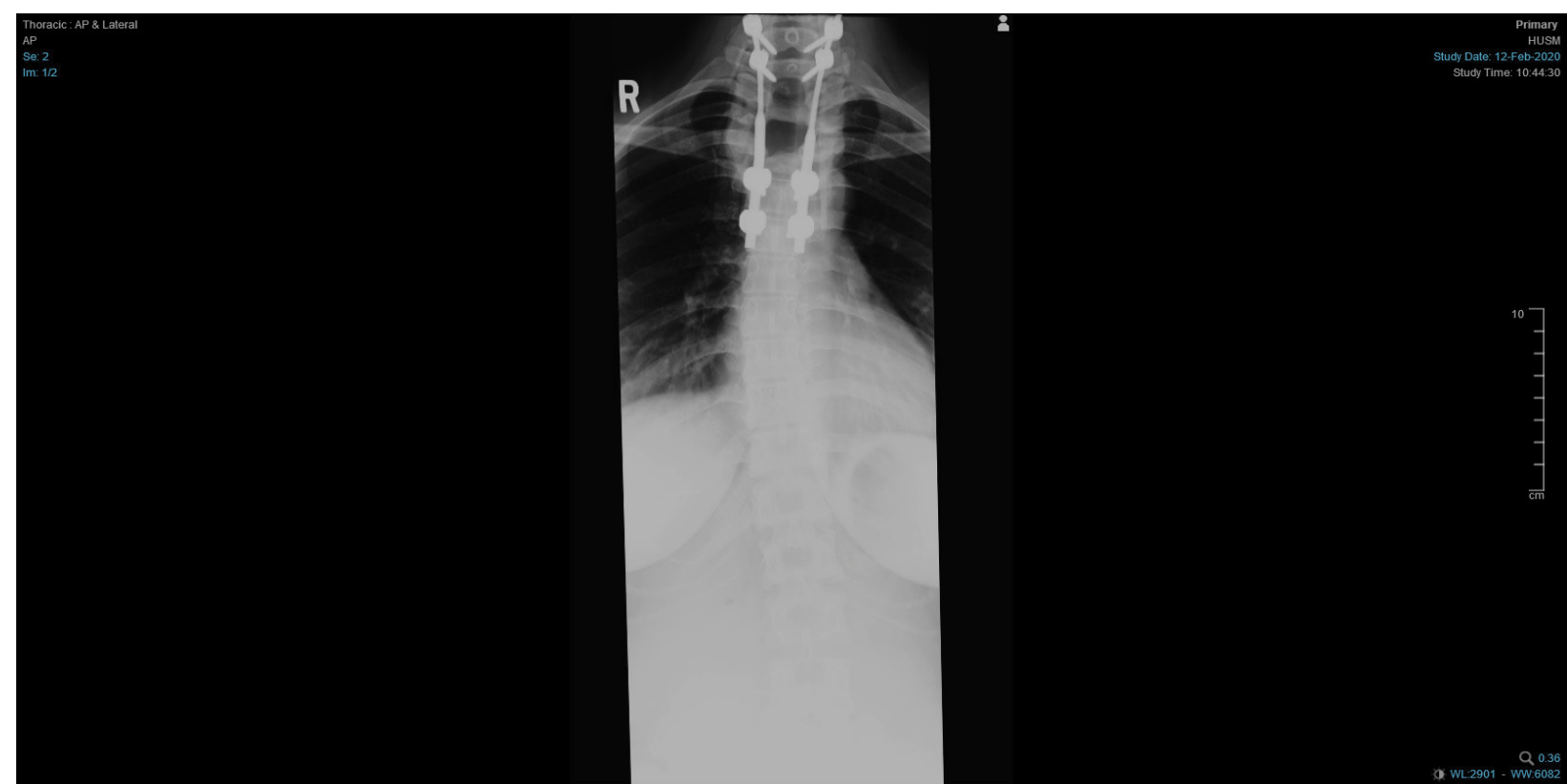

Figure 8: AP thoracic X-ray after three months showing Rod implants on both vertebral sides are intact.

seven months post-operative X-ray view.

\section{Discussion}

Although TB is an antique disease, it still has a worldwide impact, with an occurrence rate of nine million new cases each year and a mortality rate of two million deaths each year [4]. Spinal TB remains the most prevalent spine infection globally, and in cases with extensive spine involvement, vertebral body collapse, or severe deformity, surgical options may be considered more appealing, allowing for simultaneous treatment of infection and deformity. Symptom onset is frequently insidious in spinal TB, and disease progression is slow. The duration of symptoms at diagnosis ranges from two weeks to several years [4].

Spinal TB is a disease caused by Mycobacterium tu- berculosis infection, which often leads to the destruction of the involved vertebrae and progressive deformity of the vertebral alignment [5]. It accounts for nearly $50 \%$ of the cases of musculoskeletal TB [6-8]. Significant numbers of patients with spinal TB, especially in developing countries, still present late after disease onset with severe neurological deficits [9]. TB in the thoracic and lumbar regions mostly destroys the vertebral body, intervertebral disc, and surrounding soft tissue. It often leads to spinal kyphosis, spinal cord or nerve compression, and the loss of mechanical stability [10].

The indications for surgery have not changed significantly since the "middle path regimen" was proposed by Tuli in 1975 for spinal TB [4]. Surgical treatment should be considered in the following four situations if the patient [10]: 


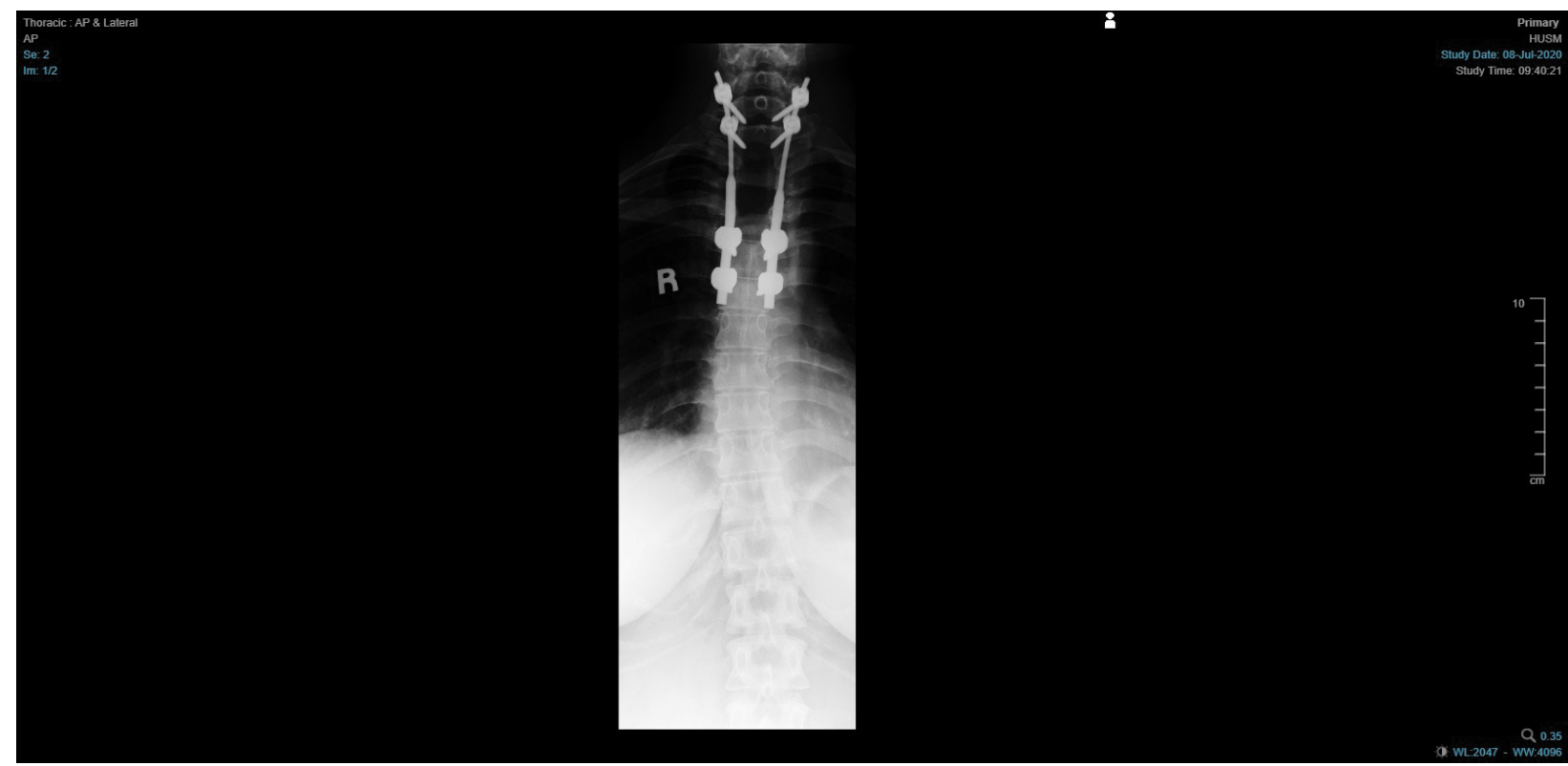

Figure 9: AP thoracic X-ray after seven months showing Rod implants on both vertebral sides are intact.

- Insensitive/resistant to anti-TB chemotherapy drugs.

- Has severe vertebral body damage.

- Has neurological dysfunction.

- Has kyphosis or spinal instability.

Different surgical approaches for various spinal lesions, these approaches exist depending on the situation of the abscess and the bone lesion [3]. For cervical spine TB, an anterior approach is most common, while in thoracic spine TB, several options are available, including anterior and anterolateral decompression by thoracoabdominal approach, and posteriorly there is costo-transversectomy and laminectomy.

Predominantly in the thoracic spine, there are more probabilities of deformity as the weight passes through the anterior column and for lumbar level spinal TB, posterior, anterior, and anterolateral are all viable options. The goals of current surgical management of spinal TB include debridement of affected vertebrae, correction of deformities, decompression of spinal cord, stabilization of the spine, and extra protection of the spinal cord [4].

Neurological involvement due to Pott's disease is quite benign if urgent decompression accomplished at the onset of the disease [11], and a significant percentage of affected patients with spinal TB and motor deficits experience remarkable improvement after surgical decompression [9].

\section{Conclusion}

Surgical intervention in a patient with spinal TB has many advantages, especially if the patient presented with neurological findings suggesting spinal cord compression and spinal deformity. The advantages include decompression of the spinal cord, debridement of af- fected vertebrae, and adequate spinal stabilization. Performing surgical intervention with fibula bone allograft holding and supporting Rod implants by acting as a firm base gives good postoperative results. It improves neurological manifestations resulting from nerve root compression due to collapsed and fused vertebrae affected by spinal TB.

\section{References}

1. Pigrau-Serrallach C, Rodríguez-Pardo D (2013) Bone and joint tuberculosis. Eur Spine J 22: 556-566.

2. Ansari S, Amanullah Md F, Ahmad K, Rauniyar RK (2013) Pott's Spine: Diagnostic imaging modalities and technology advancements. N Am J Med Sci 5: 404-411.

3. Guerado E, Cervan AM (2012) Surgical treatment of spondylodiscitis. An update. Int Orthop 36: 413-420.

4. Alam MS, Phan K, Karim R, Jonayed SA, Munir HKM, et al. (2015) Surgery for spinal tuberculosis: A multi-center experience of 582 cases. J Spine Surg 1: 65-71.

5. Yin X, Liu P, Liu Y, Fan WL, Liu B, et al. (2017) Utilization of ring-shaped bone allograft for surgical treatment of adolescent post-tubercular kyphosis: A retrospective study. Medicine 96: e7132.

6. Liu JM, Chen XY, Zhou Y, Long XH, Chen WZ, et al. (2016) Is nonstructural bone graft useful in surgical treatment of lumbar spinal tuberculosis?: A retrospective case-control study. Medicine 95: e4677.

7. Shen X, Huang X, Xiao S, Liu H, Zhang Y, et al. (2015) Surgical treatment of selected patients with multilevel contiguous thoracolumbar spinal tuberculosis by only posterior instrumentation without any bone fusion. Int J Clin Exp Med 8: 18611-18619.

8. Zhang J, He WS, Wang C, Yan YG, Ouyang ZH, et al. (2018) Application of vascularized fibular graft for reconstruction and stabilization of multilevel cervical tuberculosis: A case report. Medicine (Baltimore) 97: e9382.

9. Sai Kiran NA, Vaishya S, Kale SS, Sharma BS, Mahapatra AK, et al. (2007) Surgical results in patients with tuberculo- 
sis of the spine and severe lower-extremity motor deficits: A retrospective study of 48 patients. J Neurosurg Spine 6: 320-326.

10. Tang Y, Wu W, Yang S, Wang DG, Zhang Q, et al. (2019) Surgical treatment of thoracolumbar spinal tuberculosis-a multicentre, retrospective, case-control study. Journal of Orthopaedic Surgery and Research 14: 233.

11. Turgut M (2001) Spinal tuberculosis (Pott's disease): Its clinical presentation, surgical management, and outcome. A survey study on 694 patients. Neurosurg Rev 24: 8-13. 